\title{
Building Change Detection from Uniform Regions
}

\author{
Charles Beumier and Mahamadou Idrissa \\ Signal \& Image Centre, Royal Military Academy, 1000 Brussels, Belgium \\ \{charles.beumier, idrissa\}@elec.rma.ac.be
}

\begin{abstract}
This paper deals with building change detection by supervised classification of image regions into 'built' and 'non-built' areas. Regions are the connected components of low gradient values in a multi-spectral aerial image. Classes are learnt from spectral (colour, vegetation index) and elevation cues relatively to building polygons and non building areas as defined in the existing database. Possible candidate building regions are then filtered by geometrical features. Inconsistencies in the database with the recent image are automatically detected. Tests in cooperation with the Belgian National Geographical Institute on an area with sufficient buildings and landscape variety have shown that the system allows for the effective verification of unchanged buildings, and detection of destructions and new candidate buildings.
\end{abstract}

Keywords. Building verification, building detection, spectral cues, geometrical cues, Digital Surface Model.

\section{Introduction}

The production of cartographic or digital geographic information is currently living an important change. The ever increasing demand for up-to-date data requires the development of procedures keeping the human intervention to an acceptable level.

One traditional approach for geographical data update requires the analyst to observe aerial images and update the digital data accordingly. Recent projects (e.g. project WIPKA, Germany [1]; project MAJEC, France [2]; project ATOMI, Switzerland [3]; DB TOP10DK, Denmark [4]) try to help the analyst in localising semiautomatically plausible changes from recent aerial imagery.

Our research department has been active in change detection in remote sensing for nearly 10 years, in cooperation with the Belgian Geographical National Institute. After considering the detection of change from satellite images at the level of building blocks, the team has concentrated its effort on DSM (Digital Surface Model) extraction from stereoscopic pairs and change detection from aerial images at $0.3 \mathrm{~m}$ resolution. In this respect, the previous publication [10] described the detection of building changes from spectral cues (colour, NDVI) and local elevation.

In this paper, we present building change detection thanks to the inclusion of geometrical clues as a way to reduce false alarms and improve the detection of candidate new buildings. 


\section{Change Detection}

Conventional solutions for topographic database production generally rely on stereoscopic aerial colour images with resolution better than $50 \mathrm{~cm}$. To reduce the amount of work to keep the database up to date, only changes are looked for as the majority of data remains valid.

As confirmed by the activities about change detection organised by EuroSDR [5], National Mapping Agencies and other map suppliers are looking for (semi-) automatic solutions in order to lighten the needed human resources and shorten the update latency. Considering the bibliography about change detection for topo-geographic database update in the case of aerial images [1-8], most approaches rely on the same extracted information: colour, vegetation index and elevation (DSM and DTM, Digital Terrain Model). For instance the works of N. Champion [2] and M. Niederöst [3] both exploited a vegetation mask obtained from the multispectral data and a normalised Digital Surface Model derived from the DSM. In [2] candidate buildings are looked for thanks to DSM contours and 3D line segments, while in [3] blob detection or rectangle fitting are used to try to reconstruct a 3D building shape.

We believe that $3 \mathrm{D}$ lines are indeed a fundamental clue as a way to confirm or highlight man made structures. However we preferred first to segment the image and look for regions consistent with being a building roof. For this, colour, vegetation and elevation features are measured and averaged over the regions and compared to values encountered in building regions of the database. In comparison with our previous work [10], the pixel analysis is replaced by a region analysis, normally more robust, fast and enabling geometrical constraints to be applied. A prospective goal, not yet implemented, is the possible integration of topological clues (presence of shadow neighbouring regions, alignment of buildings and proximity of road parts).

\section{Spectral Cues}

The most direct information extracted from the multispectral image concerns the red $(\mathrm{R})$, green $(\mathrm{G})$, blue (B) and near Infrared (nIR) pixel values.

\subsection{Exploiting R, G, B, nIR}

$\mathrm{R}, \mathrm{G}$ and $\mathrm{B}$ values are highly correlated by intensity so that derived values like HSI or Lab are preferable to better discriminate objects based on colour hue and intensity. The uniformity of spectral features is a good criterion to find objects or part of them.

If buildings are quite easily delineated thanks to elevation (DSM), one common difficulty is to eliminate false targets due to vegetation (trees). The near infra-red channel allows for the computation of an index highlighting vegetated areas. 


\subsection{Shadow}

Shadow areas do not receive much of the sun illumination and are henceforth imaged with little intensity and contrast. They typically suffer from a decrease in spectral specificity, bad vegetation discrimination and errors in a DSM obtained from stereo image matching. Identifying shadow regions is appealing either to reject doubtful conclusions related to such areas or to use them as evidence for the presence of elevated object in the sun direction.

\section{Geometrical Cues}

Stereoscopic pairs of images enable ortho-rectification so that produced maps are geometrically correct. They also allow for the capture of a digital surface model (DSM) which can provide for elevation values in the vector databases.

For change detection, the DSM usually helps localising potential building candidates using the local elevation relatively to a DTM, digital model of the terrain.

Other potential geometric features concern the planar extent of elevated objects. The objective is to detect image objects having some characteristics related to size and shape. As such, a building is rather blob shaped while road segments are elongated. The difficulty is to find the real building extent because roofs are rarely uniform, possibly containing different faces with different intensities.

We propose to segment the image into regions of uniform spectral values, representing parts or whole of landscape objects like roofs, roads, water areas or fields. We prefer to design a many-to-one approach (many regions, one building object) since an object usually consists of parts with different spectral properties.

\subsection{Region Segmentation}

We handle image segmentation with the most forward, simple and fast approach that we know: connected component labelling. Typically designed for binary images, this procedure consists in assigning a unique label to all connected pixels. The algorithm first scans the image to assign a label to each pixel, propagating labels already assigned in case of connectivity. Some regions appear to be connected quite late, so their labels have to be merged in a second image scan.

The key point of this approach is to get an adequate binary image. Since we are looking for spectrally uniform areas, we propose to threshold the image based on the gradient defined by the Euclidian distance of RGB values (and not only luminance). Low threshold values are chosen since we prefer over-segmentation with regions that will be later grouped into possible objects. The threshold value is therefore not very critical and we used the percentile 80 of the histogram of gradient values. We had to include the elevation in the process since regions in shadow sometimes contain roof and ground pixels due to the lack of gradient in dark areas. We added to the gradient image the edge pixels of the normalised DSM thresholded at $2.5 \mathrm{~m}$. 


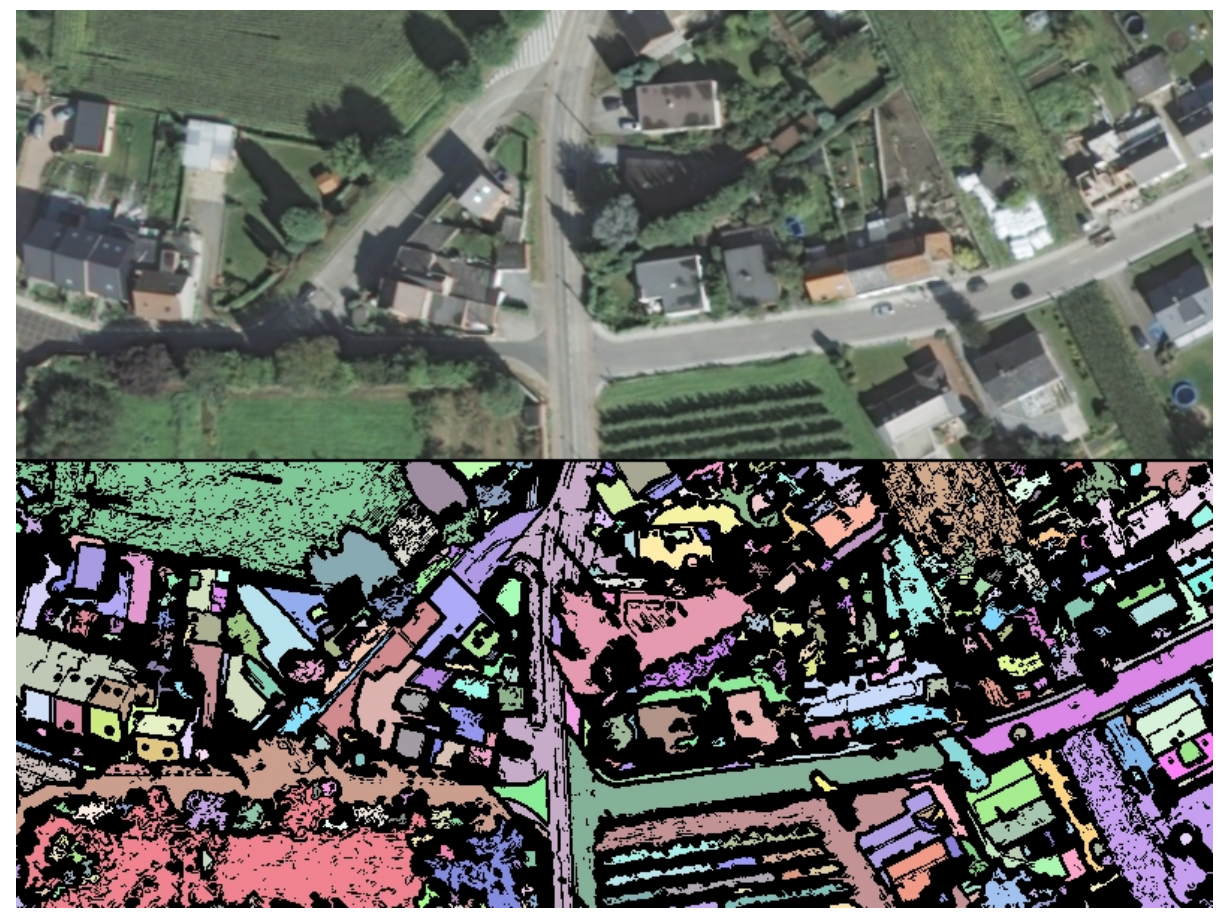

Fig. 1. Segmentation results with regions represented with random colours

For the kind of images we are working with, there are about 4000 regions (of at least 44 pixel $=4 \mathrm{~m} 2$ ) per $\mathrm{km}^{2}$.

\subsection{Digital Surface Model}

A Digital Surface Model is a collection of elevation values over a geographical area. In our department, DSMs are produced by stereo matching of image pairs with proprietary software using multiple size windows and a regularization procedure [9]. For change detection, DSMs are very powerful at highlighting potential buildings, if vegetation is filtered out (trees, woods). Since the terrain is rarely a flat surface, it has to be subtracted to the DSM to form the normalised DSM (nDSM = DSM - DTM). The nDSM is thresholded to find candidate buildings. DTMs can be derived from the DSM [2] or are available from national mapping agencies or from the SRTM campaign.

\subsection{Feature Extraction}

Each region, made of pixels with similar spectral values, is attributed average values for the luminance $\mathrm{L}((\mathrm{R}+\mathrm{G}+\mathrm{B}) / 3)$, the colour factors $\mathrm{a}^{\prime}(\mathrm{R}-\mathrm{G})$ and $\mathrm{b}^{\prime}(\mathrm{G}-\mathrm{B})$ and the vegetation index NDVI. (Mention that a' and b' are not the components of the famous L*a*b* colour space.) 
The first geometrical feature is the area of the region which allows for the filtering of tiny objects $\left(<4 \mathrm{~m}^{2}\right)$ and huge areas $\left(>2000 \mathrm{~m}^{2}\right)$.

The second geometrical clue is the average elevation $\mathrm{nZ}$ (from the normalised DSM). A $2 \mathrm{~m}$ threshold is used as garden huts possibly populate the database.

The next two geometrical values are derived from the 2D pixel distribution of the region: the extreme moments Mmin and Mmax. Their ratio Mmax/Mmin gives an idea of the elongation of the region. This ratio has been limited to 10 to reject elongated shapes like road or railway segments.

Finally, for large regions, the external outline was analysed to look for linear parts of at least $5 \mathrm{~m}$. This helps distinguishing natural objects from man-made structures.

\subsection{Discussion}

Segmentation into regions of uniform spectral values brings many advantages. First, it groups similar pixels into entities closer to the searched objects and allows for a compact representation (mean feature values). Secondly, it simplifies the computation of geometric parameters (area, elevation, elongation and outline). Finally it allows for the elimination of shadow regions which usually disturb approaches due to their poor colour content, weak NDVI estimation and erroneous DSM values.

Moreover regions will be particularly well suited in future developments to check contextual rules like the building alignment or distance and road proximity.

\section{Classification}

We handle building change detection by supervised classification of image regions into 'built' and 'non-built' classes. Since the database (to be updated) generally contains a small percentage of changes, it may be used to learn the two classes. Regions of the built class which do not satisfy geometrical constraints are then discarded.

\subsection{Learning}

Learning building likelihood from features consists in filling a 5-dimensional histogram containing the 'in' (built, according to the database) and 'out' (non-built) region counts for feature values (L, a', b', NDVI, nZ). Each feature range is divided into a few intervals based on the value distribution (regular spacing). The $\mathrm{nZ}$ and NDVI have only 2 bins as they can be handled by a threshold. L has 7 bins since its dynamic range is large while a' and b' have both 5 bins. Each histogram cell receives a feature vector likelihood based on the proportion of in and out counts of region, normalised by the global proportion for the entire set of regions. Empty cells are attributed a likelihood of -1 to label the corresponding feature vector as 'Class unknown'.

Mention that if the number of regions is small, the histogram approach is preferably replaced by nearest neighbour counting, comparing the proportion of in and out count of regions with similar feature vector. 


\subsection{Change Detection}

Each region receives a 'built up' likelihood corresponding to the 5D feature vector thanks to the histogram. For building verification (modification/destruction or 'No Change'), each DB building polygon is assigned the maximum likelihood of all the regions mainly (at least $50 \%$ of the area) contained in the polygon. For new building detection, any region with high likelihood is proposed for confirmation. The threshold on likelihood is adapted by the operator who solves the trade-off between low false alarms and low miss rate. For Fig. 2, the threshold on the likelihood was set to 0.6.

In order to reduce the number of false alarms for new candidate buildings, geometrical constraints were applied. The tiny and huge regions were discarded. The moment ratio helped removing elongated regions corresponding to road or railway segments or long shadow areas. The difficult shadow regions, having poor spectral specificity and possibly elevated, were rejected based on an intensity threshold obtained from the known building side opposite to the sun [11]. Finally, large regions whose external outline does not contain linear parts $(>5 \mathrm{~m})$ were also discarded.

According to the database (old) and image (new) classes, four cases arise: a) the building still exists (green); b) the building is destroyed/modified (blue); c) a new building appeared (red); there was never a building (transparent). We take for granted that a polygon is verified as soon as one region has sufficient likelihood. As visible on Fig. 2, many regions of gable roofs are in shadow and receive little support from spectral or geometrical clues. This explains why there are many blue areas in the results.

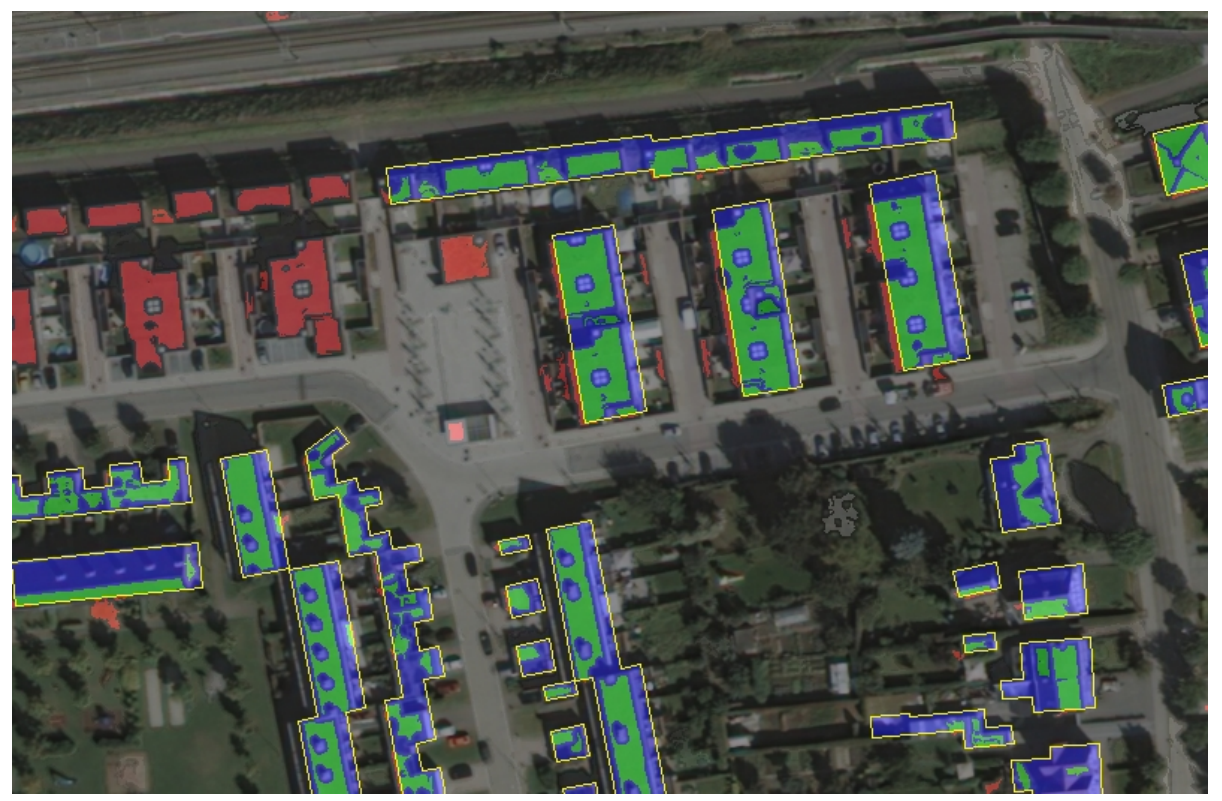

Fig. 2. Classified regions. Green for built regions mainly in building database polygon, Blue for polygon part not verified, Red for built regions not in the database. 


\subsection{Building Completion}

A last step is welcome to obtain an accurate change detection map. We noticed in Fig. 2 that many buildings are only partially covered by supporting regions. In order to detect object boundaries as close as possible to reality, the seed regions (green and red) have to be enlarged. We propose to use the normalized DSM and NDVI and create a building mask which is converted into regions by a connected component labeling. Each region is a candidate building if it contains at least a red or green classified region.

\section{Results}

A first zone representative for tests has been proposed by our client. The data set, covering $4 \mathrm{~km}^{2}$ around Leuven, consists of the database vectors as well as the RGB and Near Infrared images (stereo pairs at $0.3 \mathrm{~m}$ resolution). We produced a DSM at $0.3 \mathrm{~m}$ and extracted the regions from the RGB ortho-rectified images. We derived the spectral and geometrical features for each region.

The classification results produced by the histogram approach and geometrical constraints were displayed for quality analysis by inspection (Fig. 2 is a small part). Results displayed with 3 colours facilitate the counting of the different cases. For a region of $4 \mathrm{~km}^{2}$ containing about 1200 polygons, we observed:

Table 1. Change detection results in number of cases (database polygons or regions)

\begin{tabular}{|l|l|l|}
\hline Cases & All polygons (1164) & Polygons > 30m2 (974) \\
\hline $\begin{array}{l}\text { Unchanged buildings } \\
\text { (at least partly green) }\end{array}$ & 1012 & 927 \\
\hline Not verified (totally blue) & 141 & 47 \\
\hline New buildings (red) & 117 & 59 \\
\hline Bad New & 66 & 28 \\
\hline
\end{tabular}

Although the numbers of detected changes appear too high to relieve the operator of much image interaction, most errors are due to helpless situations or unimportant cases. We noticed several houses hidden in the woods. Many undetected buildings concern the small and low annexes or garden huts. This is secondary change information for the database provider which can hardly be dealt with the current image resolution. For that reason, we derived the statistics for building larger than $30 \mathrm{~m} 2$ as well.

In the reasonable situation focusing on large polygons $(>30 \mathrm{~m} 2)$, we obtained about $4 \%$ of bad verification, some cases of which are helpless (hidden by trees). 59 regions were detected as potential building parts, half of which were not real buildings although some of them could be considered as such (e.g. containers).

\section{Conclusions}

This paper has presented building change detection between a digital vector database and stereo aerial imagery. The colour image is segmented into regions of uniform 
colours and elevation. These regions receive attribute values for L, a', b', NDVI, local elevation (nDSM) and other geometrical features. These attributes help classifying regions with a histogram approach after a learning procedure using the a priori classes building / non-building as stated in the database to update. Many regions are discarded thanks to geometrical features. Compared to previous results, the region approach has similar verification performance for known database building polygons but reduces the number of false alarms when looking for new candidate buildings.

We intend to use the region approach to add contextual constraints like the relation between dark areas (shadow) and elevated regions, and the link between the road network and the presence of buildings.

\section{References}

1. Busch, A., Gerke, M., Grünreich, D., Heipke, C., Liedtke, C.-E., Müller, S.: Automated Verification of a Topographic Reference Dataset: System Design and Practical Results. In: Int. Archives of Photogrammetry and Remote Sensing IAPRS, Istanbul, Turkey, vol. XXXV, B2, pp. 735-740 (2004)

2. Champion, N., Stamon, G., Pierrot-Deseilligny, M.: Automatic Revision of 2D Building Databases from High Resolution Satellite Imagery: A 3D Photogrammetric Approach. In: AGILE, Hannover Germany (2009)

3. Niederöst, M.: Detection and Reconstruction of Buildings for Automated Map updating, These Institut für Geodäsie und Photogrammetrie, ETH Zürich (2003)

4. Olsen, B.: Automatic Change Detection for Validation of Digital Map Databases. In: ISPRS XX, Commission II, Istambul, vol. XXXV, pp. 569-574 (2004)

5. Heipke, C., Mooney, K.: EuroSDR - A research organisation serving Europe's geospatial information needs. In: Fritsch, D. (hrsg.) Photogrammetric Week 2009, pp. 321-330. Wichmann, Heidelberg (2009)

6. Baltsavias, E.: Object Extraction and Revision by Image Analysis using Existing Geodata and Knowledge: Current Status and Steps towards Operational Systems. ISPRS Journal of Photogrammetry and Remote Sensing 58, 129-151 (2004)

7. Matikainen, I., Hyyppa, J., Ahokas, E., Markelin, L., Kartinen, H.: Automatic Detection of Buildings and Changes in Buildings for Updating of Maps. Remote Sensing 2, 1217-1248 (2010)

8. Rottensteiner, F.: Building Change Detection from Digital Surface Models and Multispectral Images. In: Photogrammetric Image Analysis, Munich, Germany, pp. 145-150 (2007)

9. Idrissa, M., Lacroix, V.: A Multiresolution-MRF Approach for Stereo Dense Disparity Estimation. In: IEEE-GRSS/ISPRS Joint Urban Remote Sensing Event, Shanghai, China (2009)

10. Beumier, C., Idrissa, M.: Building Change Detection by Histogram Classification. In: Int. Conf. on Signal-Image Technology and Internet-Based Systems, Dijon, France (2011)

11. Beumier, C.: Building verification from geometrical and photometric cues. In: Applic. of Digital Image Processing XXX, San Diego, California. Proc. of SPIE, vol. 6696 (2007) 